\title{
Femtosecond tunneling of polarons in $\mathrm{Pb}_{5} \mathrm{Cr}_{3} \mathrm{~F}_{19}$
}

\author{
A. Levstik*, C. Filipič, G. Tavčar and B. Žemva \\ Jožef Stefan Institute, Jamova 39, 1000 Ljubljana, Slovenia \\ *adrijan.levstik@ijs.si
}

Received 21 February 2014; Accepted 15 June 2014; Published 28 July 2014

\begin{abstract}
The complex dielectric constant/ac electrical conductivity was investigated as a function of frequency and temperature in $\mathrm{Pb}_{5} \mathrm{Cr}_{3} \mathrm{~F}_{19}$. The system undergoes a ferroelectric phase transition at higher temperatures. At lower temperatures the real part of the complex ac electric conductivity was found to follow the universal dielectric response (UDR) $\sigma^{\prime} \propto \nu^{s}$, typical for hopping or tunneling of localized charge carriers. A detailed analysis of the temperature dependence of the UDR parameter $s$ in terms of the theoretical model for tunneling of small polarons revealed that, at low temperatures, this mechanism governs the charge transport in $\mathrm{Pb}_{5} \mathrm{Cr}_{3} \mathrm{~F}_{19}$. The value of the inverse attempt frequency $\tau_{0}$ indicates the femtosecond tunneling of polarons in the system similar to the BüttikerLandauer transversal time.
\end{abstract}

Keywords: Low-field transport; tunneling; polarons and electron-phonon interactions.

\section{Introduction}

In attosecond physics, experimental investigations of atomic interactions with intense laser fields have demonstrated tunneling times of electrons in different time scales from $10^{-12}$ to $10^{-18} \mathrm{~s}$, depending on electron motion on atomic or molecular scales. ${ }^{1}$

In the present paper, we show that similar tunneling times of polaronic electrons are observed from the classical experiment where tunneling time is determined via measurements of frequency and temperature dependence of dielectric constant and electrical conductivity and their analysis using the theoretical model describing small polarons. We show that polarons play an important role in magnetoelectric ${ }^{2}$ $\mathrm{Pb}_{5} \mathrm{Cr}_{3} \mathrm{~F}_{19}$ as the dominant charge transport mechanism at low temperatures.

Magnetoelectric systems are at the cutting edge of present day material science, since the possibilities of electric field control of magnetism and vice versa allow, among other things, an electric readout of magnetically stored information, magnetic capacitors, new spintronic elements and various sensors. $^{3}$

\section{Experiment}

Polycrystalline $\mathrm{Pb}_{5} \mathrm{Cr}_{3} \mathrm{~F}_{19}$ was prepared by reacting $\mathrm{CrF}_{3}$ with $\mathrm{PbF}_{2}$ in a 3:5 molar ratio. The reaction mixture was placed in a gold reaction vessel, sealed, and heated for $48 \mathrm{~h}$ at $800 \mathrm{~K}$, as described previously. ${ }^{4}$

The complex dielectric constant $\varepsilon^{*}(\nu, T)=\varepsilon^{\prime}-i \varepsilon^{\prime \prime}$ was measured between 50 and $650 \mathrm{~K}$ in the frequency range of $1 \mathrm{~Hz}$ to $1 \mathrm{MHz}$, using a Novocontrol Alpha High Resolution Dielectric Analyzer. The amplitude of the probing ac electric signal was $1 \mathrm{~V} / \mathrm{mm}$. The temperature was stabilized within $\pm 0.1 \mathrm{~K}$ using an Oxford Instruments continuous flow cryostat. The real part of the complex ac conductivity $\sigma^{*}(\nu, T)=$ $\sigma^{\prime}+i \sigma^{\prime \prime}$ was obtained from $\sigma^{\prime}=2 \pi \nu \varepsilon_{0} \varepsilon^{\prime \prime}$, with $\varepsilon_{0}$ being the permittivity of the vacuum.

\section{Results and Discussion}

$\mathrm{Pb}_{5} \mathrm{Cr}_{3} \mathrm{~F}_{19}$ is ferroelectric below $T_{C}=540 \mathrm{~K}$. The temperature dependence of the dielectric constant was measured at three frequencies (Fig. 1) and the dielectric constant reveals the ferroelectric phase transition at $540 \mathrm{~K}$. High-temperature EPR spectra supported the occurrence of the para-ferroelectric transition. A phase transition at $545 \mathrm{~K}$, where the temperature dependence of EPR susceptibility, the line width $\delta H$, and the $g$-factor show pronounced step in their values, was demonstrated by Blinc et al. ${ }^{2,5}$ An antiferro-magnetic transition was also observed with $T_{N} \sim 11 \mathrm{~K}^{2}$ Results of lowtemperature EPR spectra were confirmed by magnetic measurements. No magnetic hysteresis loops exist below $11 \mathrm{~K}$, thus excluding ferromagnetic, ferrimagnetic, and spin glass order, but the relationship between magnetization and magnetic field is not linear. The observed changes of the magnetic properties ongoing through the ferroelectric phase transition exhibit magnetoelectric coupling. ${ }^{2}$

In order to characterize $\mathrm{Pb}_{5} \mathrm{Cr}_{3} \mathrm{~F}_{19}$ at low temperatures, the temperature dependence of the complex dielectric constant/ac electrical conductivity, as a function of the frequency, was investigated in detail. The temperature dependence of the real part of the complex ac conductivity, $\sigma^{\prime}$, in $\mathrm{Pb}_{5} \mathrm{Cr}_{3} \mathrm{~F}_{19}$, was measured at several frequencies (Fig. 2). Below $300 \mathrm{~K}, \sigma^{\prime}$ is seen to be almost independent of temperature till $10 \mathrm{~K}$ while,

This is an Open Access article published by World Scientific Publishing Company. It is distributed under the terms of the Creative Commons Attribution 3.0 (CC-BY) License. Further distribution of this work is permitted, provided the original work is properly cited. 


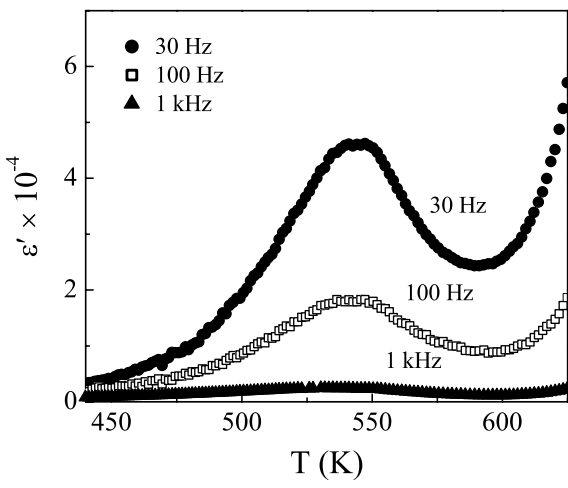

Fig. 1. Temperature dependence of the dielectric constant in $\mathrm{Pb}_{5} \mathrm{Cr}_{3} \mathrm{~F}_{19}$, measured at three frequencies.

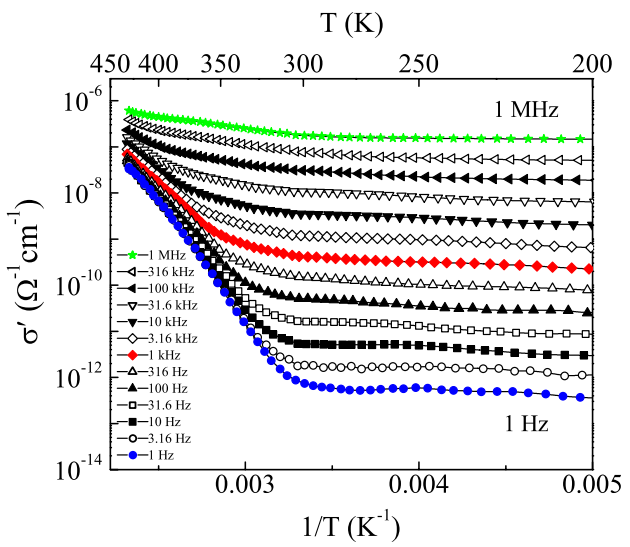

Fig. 2. Temperature dependence of the real part of the complex ac electrical conductivity, $\sigma^{\prime}$, in $\mathrm{Pb}_{5} \mathrm{Cr}_{3} \mathrm{~F}_{19}$, measured at several frequencies.

at higher temperatures, it becomes strongly temperature dependent. Moreover, at higher temperatures still, with reduced measuring frequency, $\sigma^{\prime}$ tends towards linear dependence, suggesting that the dc conductivity might reveal thermally activated behavior. The strongly frequency-dependent plateaus in $\sigma^{\prime}(\mathrm{T})$ below $300 \mathrm{~K}$ suggest that hopping or tunneling of localized charge carriers governs the electrical transport at lower temperatures. ${ }^{6}$

The temperature dependence of the ac electrical conductivity, $\sigma^{\prime}$, measured at $1 \mathrm{~Hz}$, (Fig. 3 ) is close to that of the dc conductivity. $\operatorname{Ln}\left(\sigma^{\prime}\right)$ tends towards a linear dependence on $1 / T$, therefore the activation energy, $E_{\mathrm{dc}}$, was determined from the slope using the equation for hopping of small polarons. ${ }^{7,8}$

Polarons are best identified in measurements with charge carriers in nonmetals or doped semiconductors. ${ }^{9}$ The concept of polarons can represent the basis for understanding the complex physical properties of strongly correlated electronic systems exhibiting high-temperature superconductivity or the colossal magnetoresistance. ${ }^{10}$ In fact, besides the occurrence of mid-infrared excitation in the optical conductivity, ${ }^{11,12}$ the fingerprint of polarons is usually associated with the temperature and frequency dependence of the ac conductivity.

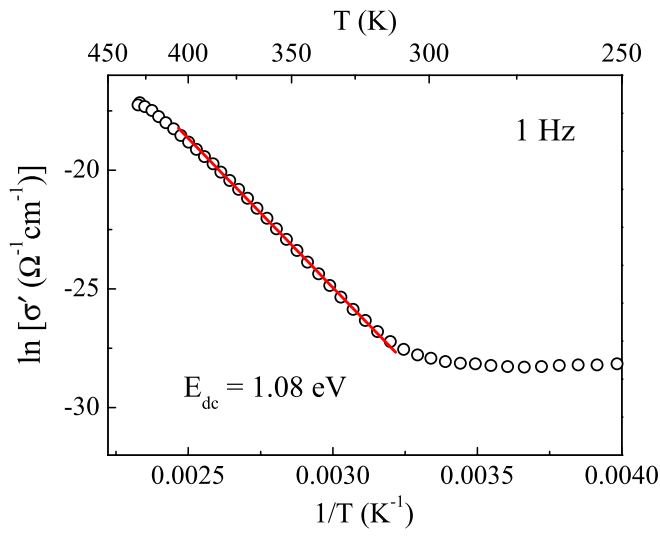

Fig. 3. $\operatorname{Ln}\left(\sigma^{\prime}\right)$, measured at $1 \mathrm{~Hz}$, as a function of the inverse temperature.

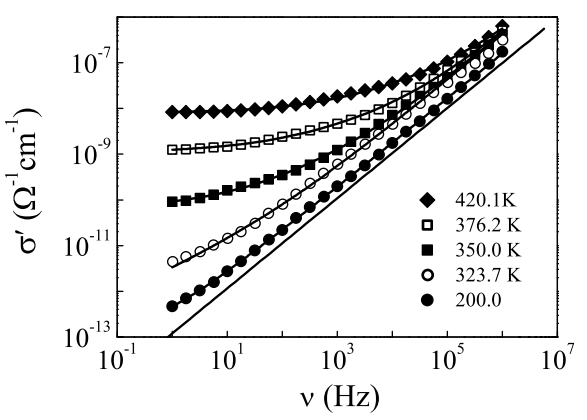

(a)

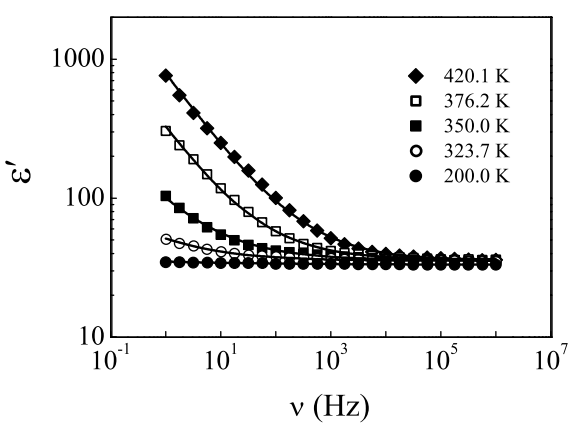

(b)

Fig. 4. (a) Frequency dependence of the real part of the complex electrical conductivity, $\sigma^{\prime}$, in $\mathrm{Pb}_{5} \mathrm{Cr}_{3} \mathrm{~F}_{19}$ at five selected temperatures. The solid lines are fits, performed simultaneously, for $\sigma^{\prime}(\nu)$ and $\varepsilon^{\prime}(\nu)$ to an expression combining a dc contribution, a UDR hopping contribution, a $\nu^{n}$ power law, and a limiting high frequency dielectric constant $\varepsilon_{\infty}$. The straight line indicates the slope with $n=1$. (b) Frequency dependence of the real part of the complex dielectric constant, $\varepsilon^{\prime}$, at the same temperatures as above. The solid lines are fits, performed simultaneously for $\sigma^{\prime}(\nu)$ and $\varepsilon^{\prime}(\nu)$, with the same contributions as in (a).

The frequency dependence of the ac electrical conductivity in $\mathrm{Pb}_{5} \mathrm{Cr}_{3} \mathrm{~F}_{19}$ was measured at five selected temperatures (Fig. 4(a)). At lower frequencies, $\sigma^{\prime}(\nu)$ starts to saturate towards the dc conductivity. At higher frequencies, $\sigma^{\prime}$ follows 
a power law $\sigma^{\prime}=\sigma_{2} \nu^{n}$ with $n \approx 1$ and, at the lowest temperatures, this behavior was observed over a broad frequency range of four decades. It is however known ${ }^{13}$ that the simple ansatz $\sigma^{\prime}(\nu)=\sigma_{\mathrm{dc}}+\sigma_{2} \nu^{n}$ cannot describe $\sigma^{\prime}(\nu)$ properly over the broad frequency range studied here. Figure 4(b) shows the frequency dependence of the real part of the dielectric constant at five selected temperatures as before. At higher frequencies, $\varepsilon^{\prime}(\nu)$ starts to saturate towards the highfrequency dielectric constant $\varepsilon_{\infty}$.

For many amorphous and crystal systems it has been shown that, besides the dc contribution of conductivity, $\sigma_{\mathrm{dc}}$, the frequency-dependent real part of complex ac conductivity, $\sigma^{\prime}$, follows the so-called universal dielectric response (UDR). An additional sub-linear term, $\sigma^{\prime}=\sigma_{1} \nu^{s}$ with $s \leq 1$, was therefore introduced. The UDR contribution has been predicted by various models ${ }^{14,15}$ and is the most common approach to take into account the hopping conductivity of localized charge carriers.

Under these assumptions, complex conductivity $\sigma^{*}(\nu)=$ $\sigma^{\prime}(\nu)+\sigma^{\prime \prime}(\nu)$ is given by ${ }^{16,17}$

$$
\begin{aligned}
\sigma^{\prime}(\nu) & =\sigma_{\mathrm{dc}}+\sigma_{1} \nu^{s}, \\
\sigma^{\prime \prime}(\nu) & =\tan (s \pi / 2) \sigma_{1} \nu^{s}=\nu \varepsilon_{0} \varepsilon^{\prime}(\nu) .
\end{aligned}
$$

Here, $\sigma_{1}$ is a temperature-dependent constant, $\nu$ is the measuring frequency, $s$ the frequency exponent, $s \leq 1$, and $\varepsilon_{\infty}$ being permittivity of the vacuum. From conductivity, the complex dielectric permittivity can be calculated by $\varepsilon^{*}(\nu)=$ $\varepsilon^{\prime}(\nu)-i \varepsilon^{\prime \prime}(\nu)=i \sigma^{*}(\nu) / \nu \varepsilon_{0}$.

The expression for the frequency dependence of the dielectric constant, $\varepsilon^{\prime}(\nu)=\varepsilon_{\infty}+\varepsilon_{1} \nu^{s-1}+\varepsilon_{2} \nu^{n-1}$, is obtained by the Kramers-Kronig transformation, therefore for $n=1$

$$
\varepsilon_{1} / \sigma_{1}=\tan (\pi s / 2) / \varepsilon_{0} .
$$

The above equation connects parameters $\varepsilon_{1}$ and $\sigma_{1}$, and limits the simultaneous fits of $\sigma^{\prime}(\nu)$ and $\varepsilon^{\prime}(\nu)$.

From equations, for $\sigma^{\prime}(\nu)=\sigma_{\mathrm{dc}}+\sigma_{1} \nu^{s}+\sigma_{2} \nu^{n}$ and $\varepsilon^{\prime}(\nu)=\varepsilon_{\infty}+\varepsilon_{1} \nu^{s-1}+\varepsilon_{2} \nu^{n-1}$, we derive (for $n=1$ )

$$
\begin{aligned}
& \sigma^{\prime}(\nu)=\sigma_{\mathrm{dc}}+\sigma_{1} \nu^{s}+\sigma_{2} \nu, \\
& \varepsilon^{\prime}(\nu)=\varepsilon_{\infty}+\left[\sigma_{1} \tan (s \pi / 2) / \varepsilon_{0}\right] \nu^{s-1},
\end{aligned}
$$

which we use for simultaneous fitting of $\sigma^{\prime}(\nu)$ and $\varepsilon^{\prime}(\nu)$ data. The solid lines in Figs. 4(a) and 4(b) were obtained by simultaneous fits of the experimental $\sigma^{\prime}(\nu)$ and $\varepsilon^{\prime}(\nu)$ data to the above expressions. To justify the choice $n=1$ we added the limiting line, $\sigma^{\prime} \propto \nu^{n}$ with $n=1$, to Fig. 4(a).

Figure 5 shows the temperature dependence of the exponent $s$ for $\mathrm{Pb}_{5} \mathrm{Cr}_{3} \mathrm{~F}_{19}$. The UDR exponent $s$, which was determined by the above mentioned fitting procedure, shows nonmonotonous temperature dependence with a minimum at approximately $360 \mathrm{~K}$. Such behavior is predicted by the tunneling polaron model ${ }^{14,15,18}$ (originally this model was developed for tunneling of overlapping large polarons, ${ }^{14,15} \mathrm{but}$ it was argued later ${ }^{18}$ that it is in fact applicable to small polarons), which gives the temperature dependence of $s$ as ${ }^{14,15}$

$$
s=1-\frac{4+6 W_{\infty} r_{0}^{\prime} /\left(k T R^{\prime 2}\right)}{R^{\prime}\left[1+W_{\infty} r_{0}^{\prime} /\left(k T R^{\prime 2}\right)\right]^{2}},
$$

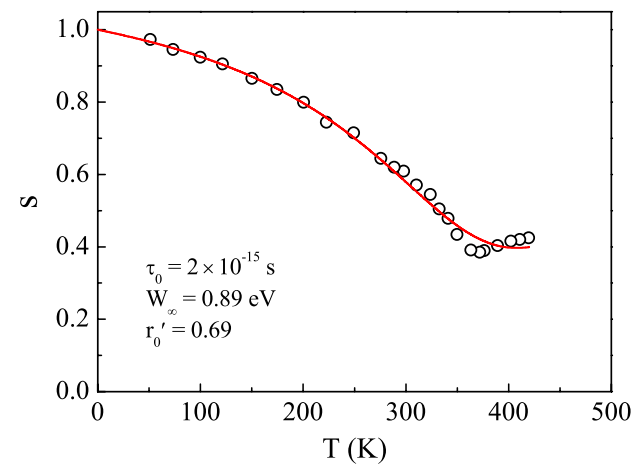

Fig. 5. Temperature dependence of the UDR parameter $s$, for $\mathrm{Pb}_{5} \mathrm{Cr}_{3} \mathrm{~F}_{19}$, determined from the simultaneous fits of the experimental $\sigma^{\prime}(\nu)$ and $\varepsilon^{\prime}(\nu)$ data (as presented in Fig. 4). The solid line is the fit to a model for small polaron tunneling [Eqs. (1) and (2)].

where $W_{\infty}$ denotes the energy barrier and $r_{0}^{\prime}$ the reduced polaron radius. The reduced tunneling distance $R^{\prime}$ is also a function of $W_{\infty}$ and $r_{0}^{\prime}$ and, additionally, of the inverse attempt frequency $\tau_{0}$ as ${ }^{14,15}$

$$
\begin{aligned}
2 R^{\prime}= & \ln \frac{1}{\omega \tau_{0}}-\frac{W_{\infty}}{k T} \\
& +\left[\left(\ln \frac{1}{\omega \tau_{0}}-\frac{W_{\infty}}{k T}\right)^{2}+\frac{4 r_{0}^{\prime} W_{\infty}}{k T}\right]^{1 / 2},
\end{aligned}
$$

where $\omega=2 \pi \nu$. We are aware that the model predicts a frequency dependence of $s$ and therefore equation $\sigma^{\prime}(\nu)=$ $\sigma_{\mathrm{dc}}+\sigma_{1} \nu^{s}$ should no longer be valid in a strong sense, i.e., with $s=$ constant. However, in the present data a pure $\nu^{s}$ contribution is seen only over a relatively small frequency range and it can be assumed that the rather weak $\ln (\nu)$ dependence of $R^{\prime}$ leads to only small deviations from Eq. (1). Fitting the $s(T)$ data to Eqs. (1) and (2) (denoted by the solid line in Fig. 5) yields values of $W_{\infty}=0.89 \mathrm{eV}, r_{0}^{\prime}=0.69$, and $\tau_{0}=2 \times 10^{-15} \mathrm{~s}$.

From the detected $\sigma^{\prime}(\nu, T), \varepsilon^{\prime}(\nu, T)$, and $s(T)$ values we can conclude that tunneling of polarons is the dominating charge transport mechanism in $\mathrm{Pb}_{5} \mathrm{Cr}_{3} \mathrm{~F}_{19}$ at temperatures below $420 \mathrm{~K}$.

In the model, in which tunneling of polarons is the dominant mechanism, an overlap of regions of lattice distortion is important. ${ }^{14}$ The height of the effective barrier, $W$, depend on the tunneling distance $(R)$ and radius of the polaron $\left(r_{0}\right): W=W_{\infty}\left(1-r_{0} / R\right)$, where the polaron energy is $W_{\infty}=e^{2} /\left(4 \pi \varepsilon_{0} r_{0} \varepsilon_{p}\right)$ and $\varepsilon_{p}^{-1}=\varepsilon_{\infty}^{-1}-\varepsilon_{s}^{-1}$, where $\varepsilon_{\infty}$ is the high frequency and $\varepsilon_{s}$ static dielectric constant.

The dielectric relaxation connected with polarons can be analyzed with the electrical modulus picture ${ }^{19}$ in order to determine the high frequency and static dielectric constants. An electric modulus $M^{*}$ is defined as the reciprocal of dielectric constant ${ }^{19} \varepsilon^{*}$

$$
M^{*}=1 / \varepsilon^{*}=M^{\prime}(\nu)+\mathrm{i} M^{\prime \prime}(\nu),
$$




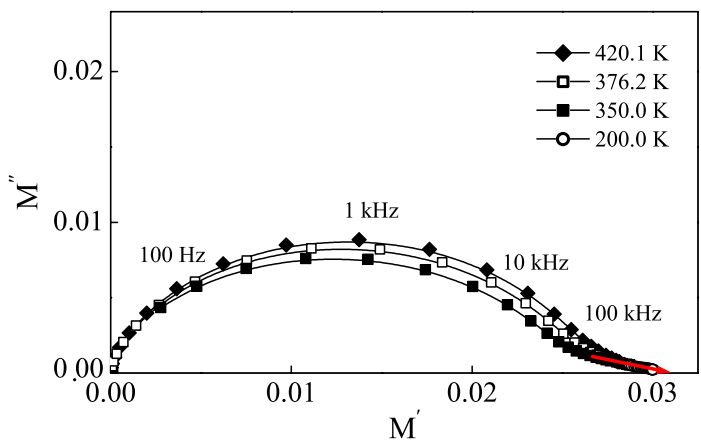

Fig. 6. (Color online) $M^{\prime \prime}$ versus $M^{\prime}$ at four temperatures in $\mathrm{Pb}_{5} \mathrm{Cr}_{3} \mathrm{~F}_{19}$. The solid lines serve as a guide for the eye. $M_{\infty}$ is extrapolated by the red straight line at high frequencies.

where

$$
M^{\prime}(\nu)=\frac{\varepsilon^{\prime}(\nu)}{\left(\varepsilon^{\prime}(\nu)\right)^{2}+\left(\varepsilon^{\prime \prime}(\nu)\right)^{2}}
$$

and

$$
M^{\prime \prime}(\nu)=\frac{\varepsilon^{\prime \prime}(\nu)}{\left(\varepsilon^{\prime}(\nu)\right)^{2}+\left(\varepsilon^{\prime \prime}(\nu)\right)^{2}}
$$

are the real and imaginary part of complex modulus, respectively.

$M^{\prime \prime}$ versus $M^{\prime}$ at four temperatures in $\mathrm{Pb}_{5} \mathrm{Cr}_{3} \mathrm{~F}_{19}$ is presented in Fig. 6. From the Cole-Cole diagram it is possible to determine $M_{\infty}^{\prime}=0.031$ at high frequencies, and $M_{s}^{\prime}$, which approaches close to zero, at low frequencies.

From the values for $M_{\infty}^{\prime}=1 / \varepsilon_{\infty}$ and $M_{s}^{\prime}=1 / \varepsilon_{s}$ we can determine the value of the polaron radius $r_{0}$ from the expressions $W_{\infty}=e^{2} /\left(4 \pi \varepsilon_{0} r_{0} \varepsilon_{p}\right)$ and $\varepsilon_{p}^{-1}=\varepsilon_{\infty}^{-1}-\varepsilon_{s}^{-1}$. The coefficient $\alpha$ can also be determined using the relation ${ }^{14}$ that connects the reduced polaron radius and the polaron radius $\left(r_{0}^{\prime}=2 \alpha r_{0}\right)$. The values of fitting parameters for $\mathrm{Pb}_{5} \mathrm{Cr}_{3} \mathrm{~F}_{19}, \mathrm{Mn}_{1}$ (38.9LiI-1.0AgI-60B $\left.\mathrm{B}_{2} \mathrm{O}_{3}: 0.1 \mathrm{MnO}\right),{ }^{20}$ and for $\mathrm{A}_{6}\left(6 \mathrm{~B}_{2} \mathrm{O}_{3}-34 \mathrm{Fe}_{2} \mathrm{O}_{3}-60 \mathrm{P}_{2} \mathrm{O}_{5}\right),{ }^{21}$ previously determined, are given in Table 1. In the last column, the oscillation period of an electron ${ }^{1} T_{\mathrm{osc}}=2 \pi(\hbar / \Delta W)$ is given if we take $\Delta W \sim W_{\infty}$. Reciprocal attempt frequency of a polaron can be compared with the oscillation period of an electron and with the Büttiker-Landauer transversal time which is determined $^{22}$ for electrons in helium between $0.45 \times 10^{-15}$ and $0.56 \times 10^{-15} \mathrm{~s}$.

Table 1. Polaron parameters of samples.

\begin{tabular}{llllllll}
\hline Sample & $\begin{array}{c}W_{\infty} \\
(\mathrm{eV})\end{array}$ & $r_{0}^{\prime}$ & $\begin{array}{c}r_{0} \\
(\AA)\end{array}$ & $\begin{array}{c}\tau_{0} \\
(\mathrm{~s})\end{array}$ & $\alpha\left(\AA^{-1}\right)$ & $\begin{array}{c}E_{\mathrm{dc}} \\
(\mathrm{eV})\end{array}$ & $\begin{array}{c}T_{\text {osc }} \\
(\mathrm{s})\end{array}$ \\
\hline $\mathrm{Pb}_{5} \mathrm{Cr}_{3} \mathrm{~F}_{19}$ & 0.89 & 0.69 & 0.51 & $2 \times 10^{-15}$ & 0.68 & 1.08 & $1.2 \times 10^{-14}$ \\
$\mathrm{Mn}_{1}$ (Ref. 20) & 1.1 & 0.50 & 1.06 & $2 \times 10^{-15}$ & 0.24 & 0.88 & $0.96 \times 10^{-14}$ \\
$\mathrm{~A}_{6}$ (Ref. 21) & 0.92 & 0.92 & 0.84 & $2 \times 10^{-16}$ & 0.55 & 0.95 & $1.1 \times 10^{-14}$ \\
\hline
\end{tabular}

\section{Conclusion}

In conclusion, the dielectric constant and ac electrical conductivity in $\mathrm{Pb}_{5} \mathrm{Cr}_{3} \mathrm{~F}_{19}$ were investigated over broad frequency and temperature ranges. The ferroelectric phase transition was observed at high temperature $(540 \mathrm{~K})$. The changes in magnetic properties observed at the ferroelectric phase transition provide evidence for a magnetoelectric coupling. At low temperatures the real part of the complex ac electric conductivity was found to follow the universal dielectric response $\sigma^{\prime} \propto \nu^{s}$, being typical for hopping or tunneling of localized charge carriers. A detailed analysis of the temperature dependence of the UDR parameter $s$ in terms of the theoretical model for tunneling of small polarons revealed that, at low temperatures, this mechanism governs the charge transport in this system. We compared the polaronic parameters of three systems $\mathrm{Pb}_{5} \mathrm{Cr}_{3} \mathrm{~F}_{19}, \mathrm{Mn}_{1}$, and $\mathrm{A}_{6}$. The reciprocal attempt frequency has values from $2 \times$ $10^{-15}$ to $2 \times 10^{-16} \mathrm{~s}$. Comparison, of these values with those for the oscillation period for electrons and for the BüttikerLandauer transversal time for electrons, shows that there is good agreement.

\section{Acknowledgments}

Research was supported by the Slovenian Research Agency (P1-0125). We acknowledge V. Bobnar for high temperature dielectric measurements.

\section{References}

${ }^{1}$ F. Krausz and M. Ivanov, Attosecond physics, Rev. Mod. Phys. 81, 163 (2009).

${ }^{2} \mathrm{R}$. Blinc et al., Magnetism in multiferroic $\mathrm{Pb}_{5} \mathrm{Cr}_{3} \mathrm{~F}_{19}$, Phys. Rev. $B$ 85, 054419 (2012).

${ }^{3}$ K. F. Wang, J.-M. Liu and Z. F. Ren, Multiferroicity: The coupling between magnetic and polarization orders, Adv. Phys. 58, 321 (1989).

${ }^{4}$ S. Arquis-Canouet, J. Ravez and S. C. Abrahams, Phase transition at $555 \mathrm{~K}$ in nonlinear optic $\mathrm{Pb}_{5} \mathrm{Cr}_{3} \mathrm{~F}_{19}$, J. Appl. Cryst. 19, 374 (1986).

${ }^{5}$ J. F. Scott and R. Blinc, Multiferroic magnetoelectric fluorides: Why are there so many magnetic ferroelectrics? J. Phys.: Condens. Matter 23, 113202 (2011).

${ }^{6}$ R. D. Gould and A. K. Hasan, A.c. electrical properties of thermally evaporated thin films of copper phthalocyanine, Thin Solid Films 223, 334 (1993).

${ }^{7}$ N. F. Mott, Conduction in glasses containing transition metal ions, J. Non-Cryst. Solids 1, 1 (1968).

${ }^{8}$ I. G. Austin and N. F. Mott, Polarons in crystalline and noncrystalline materials, Adv. Phys. 18, 41 (1969).

${ }^{9}$ N. F. Mott and E. A. Davis, Electronic Processes in Non-Crystalline Materials (Oxford University Press, New York, 1979).

${ }^{10} \mathrm{~S}$. V. Varyukhin and O. E. Parfenov, Polaron conductivity mechanism in $\mathrm{PrBa}_{2} \mathrm{Cu}_{3} \mathrm{O}_{6.9}$, JETP Lett. 58, 830 (1993). 
${ }^{11}$ D. Emin, Optical properties of large and small polarons and bipolarons, Phys. Rev. B 48, 13691 (1993).

${ }^{12}$ C. Hartinger, F. Mayr, A. Loidl and T. Kopp, Polaronic excitations in colossal magnetoresistance manganite films, Phys. Rev. B 73, 024408 (2006).

${ }^{13}$ J. Sichelschmidt, M. Paraskevopoulos, T. Brando, R. Wehn, D. Ivannikov, F. Mayr, K. Pucher, J. Hemberger, A. Pimenov, H.-A. Krug von Nidda, P. Lunkenheimer, V. Yu. Ivanov, A. A. Mukhin, A. M. Balbashov and A. Loidl, Magnetic, electronic, dielectric and optical properties of $\operatorname{Pr}(\mathrm{Ca}: \mathrm{Sr}) \mathrm{MnO}_{3}$, Eur. Phys. J. B 20, 7 (2001).

${ }^{14}$ A. R. Long, Frequency-dependent loss in amorphous semiconductors, Adv. Phys. 31, 553 (1982).

${ }^{15} \mathrm{~S}$. R. Elliott, A.c. conduction in amorphous chalcogenide and pnictide semiconductors, Adv. Phys. 36, 135 (1987).

${ }^{16}$ V. Bobnar, P. Lunkenheimer, J. Hemberger, F. Lichtenberg, J. Mannhartand and A. Loidl, Dielectric properties and charge transport in the $(\mathrm{Sr}, \mathrm{La}) \mathrm{NbO}_{3.5-x}$ system, Phys. Rev. B 65, 155115 (2002).
${ }^{17}$ P. Lunkenheimer, V. Bobnar, A. V. Pronin, A. I. Ritus, A. A. Volkov and A. Loidl, Origin of apparent colossal dielectric constants, Phys. Rev. B 66, 052105 (2002).

${ }^{18}$ M. P. J. van Staveren, H. B. Brom and L. J. de Jongh, Metalcluster compounds and universal features of the hopping conductivity of solids, Phys. Rep. 208, 1 (1991).

${ }^{19}$ H. S. Müser and J. Petersson, Thermodynamic Theory of Relaxation Phenomena, Fortschr. Phys. 19, 559 (1971).

${ }^{20}$ C. Filipič, A. Moguš-Milanković, L. Pavić, K. Srilatha, N. Veeraiah and A. Levstik, Polaronic behavior of MnO doped LiI-AgI$\mathrm{B}_{2} \mathrm{O}_{3}$ glass, J. Appl. Phys. 112, 073705 (2012).

${ }^{21}$ C. Filipič, A. Moguš-Milanković, L. Pavić, M. Karabulut and A. Levstik, Polarons in boron doped iron phosphate glasses, J. NonCryst. Solids 358, 2793 (2012).

${ }^{22}$ P. Eckle, A. N. Pfeiffer, C. Cirelli, A. Staudte, R. Dörner, H. G. Muller, M. Büttiker and U. Keller, Attosecond ionization and tunneling delay time measurements in helium, Science 322, 1525 (2008). 